$$
\text { Pontifícia }_{\substack{\text { Universidade } \\ \text { Do Rio de Janeiro }}}
$$

Dário Augusto Borges Oliveira

\title{
Segmentação e Visualização do Fígado a partir de Imagens de Tomografia Computadorizada
}

\section{Dissertação de Mestrado}

Dissertação apresentada como requisito parcial para obtenção do grau de Mestre pelo Programa de Pósgraduação em Engenharia Elétrica do Departamento de Engenharia Elétrica da PUC-Rio.

Orientador: Prof. Raul Queiroz Feitosa Co-orientador: Prof. Mauro Monteiro Correia 


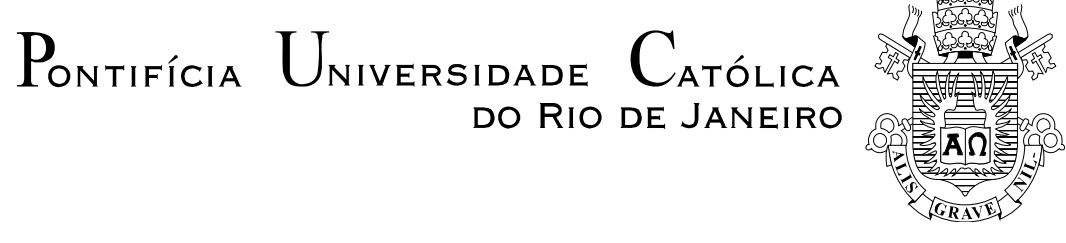

Dário Augusto Borges Oliveira

\title{
Segmentação e Visualização do Fígado a partir de Imagens de Tomografia Computadorizada
}

\begin{abstract}
Dissertação apresentada como requisito parcial para obtenção do grau de Mestre pelo Programa de Pós-Graduação em Engenharia Elétrica do Departamento de Engenharia Elétrica do Centro Técnico Científico da PUC-Rio. Aprovada pela Comissão Examinadora abaixo assinada.
\end{abstract}

Dr. Raul Queiroz Feitosa Orientador Departamento de Engenharia Elétrica - PUC-Rio

Dr. Mauro Monteiro Correia Co-Orientador INCA

Dr. Paulo Cezar Pinto Carvalho IMPA

Dr. Carlos Eduardo Thomaz Centro Universitário da FEI

Dra. Fernanda Freire Tovar Moll Redes Labs - D'OR

Prof. José Eugenio Leal Coordenador Setorial do Centro

Técnico Científico

Rio de Janeiro, 13 de abril de 2009 
Todos os direitos reservados. É proibida a reprodução total ou parcial do trabalho sem autorização da universidade, do autor e do orientador.

\section{Dário Augusto Borges Oliveira}

É formado em Engenharia Elétrica pela Universidade do Estado do Rio de Janeiro, e atualmente é aluno de mestrado no programa de Engenharia Elétrica da PUC-Rio. Têm experiência na área de processamento de imagens, atuando principalmente na detecção de padrões e construção de modelos de conhecimento com aplicações em imagens médicas e sensoriamento remoto.

Ficha Catalográfica

Oliveira, Dário Augusto Borges

Segmentação e visualização do fígado a partir de imagens de tomografia computadorizada / Dário Augusto Borges Oliveira ; orientador: Raul Queiroz Feitosa; co-orientador: Mauro Monteiro Correia. - 2009.

$78 \mathrm{f.} ; 30 \mathrm{~cm}$

Dissertação (Mestrado em Engenharia Elétrica) Pontifícia Universidade Católica do Rio de Janeiro, Rio de Janeiro, 2009.

Inclui bibliografia

1. Engenharia elétrica - Teses. 2. Imagens médicas. 3. Segmentação. 4. Level sets. 5. Algoritmos genéticos. 6. Tomografia computadorizada. I. Feitosa, Raul Queiroz. II. Correia, Mauro Monteiro. III. Pontifícia Universidade Católica do Rio de Janeiro. Departamento de Engenharia Elétrica. IV. Título. 


\section{Agradecimentos}

Agradeço a todos que contribuíram direta ou indiretamente para a realização deste trabalho, amigos, colegas e professores, e em especial:

Aos meus pais Dário e Tânia pelo suporte e amor que me formaram e fizeram de mim quem sou.

Às minhas irmãs Mariana, Silvia e Elisa, por serem quem são pra mim.

Aos meus orientadores Professores Raul Queiroz Feitosa e Mauro Monteiro Correia pelo apoio e incentivo para a realização deste trabalho.

Ao CNPq e à PUC-Rio, pelos auxílios concedidos, sem os quais este trabalho não poderia ter sido realizado.

Aos meus amigos e colegas da PUC-Rio, UERJ e CPII pela amizade e por sempre me mostrarem coisas boas da vida. 


\section{Resumo}

Oliveira, Dário Augusto Borges Oliveira; Feitosa, Raul Queiroz (Orientador); Correia, Mauro Monteiro (Co-orientador). Segmentação e visualização do fígado a partir de imagens de tomografia computadorizada. Rio de Janeiro, 2009. 78p. Dissertação de Mestrado Departamento de Engenharia Elétrica, Pontifícia Universidade Católica do Rio de Janeiro.

Esta dissertação apresenta o desenvolvimento e os resultados deste projeto de mestrado, cujo objetivo, de caráter multidisciplinar, foi desenvolver uma metodologia e uma ferramenta para segmentação do fígado, seus vasos e subregiões a partir de imagens de tomografia computadorizada da região abdominal, utilizando procedimentos de segmentação automática de imagens e visualização tridimensional de dados. A metodologia sugerida segmenta primeiramente o fígado, utilizando uma abordagem de modelos deformáveis implícitos, chamada level sets, estimando os seus parâmetros através do uso de algoritmos genéticos. Inicialmente, o contorno do fígado é manualmente definido em um tomo como solução inicial, e então o método segmenta automaticamente o fígado em todos os outros tomos, sequencialmente. Os vasos e nódulos do fígado são então identificados utilizando um modelo de mistura de funções proporcionais a gaussianas, e um método de segmentação de crescimento de regiões por histerese. As veias hepáticas e portas são classificadas dentro do conjunto de vasos, e utilizadas em uma modelagem matemática que finalmente divide o fígado em oito sub-regiões de Couinaud. Esta metodologia foi testada em 20 diferentes exames e utilizando cinco diferentes medidas de performance, e os resultados obtidos confirmam o potencial do método. Casos com baixo desempenho são apresentados para promover desenvolvimentos futuros.

\section{Palavras-chave}

Imagens Médicas. Segmentação. Level Sets. Algoritmos Genéticos. Tomografia Computadorizada. 


\section{Abstract}

Oliveira, Dário Augusto Borges Oliveira; Feitosa, Raul Queiroz (Advisor); Correia, Mauro Monteiro (Co-advisor). Liver segmentation and visualization from computer tomography images. Rio de Janeiro, 2009. 78p. MSc Dissertation - Departamento de Engenharia Elétrica, Pontifícia Universidade Católica do Rio de Janeiro.

This dissertation presents the development and results of this M.Sc project, whose multidisciplinary objective, was to develop a methodology and a tool to segment the liver, its vessels and subregions from abdominal computed tomography images, using procedures of automatic image segmentation and visualization of three-dimensional data. The suggested methodology segments initially the liver, using an approach based on implicit deformable models, called level sets, estimating its parameters using genetic algorithms. Initially, the liver boundary is manually set in one slice an initial solution, and then the method automatically segments the liver in all other slices, sequentially. Then the vessels and nodules of the liver are identified using both a model of mixture of functions proportional to Gaussians, and a segmentation method called region growing that uses hysteresis information. The hepatic and portal veins are classified within the set of vessels, and used in a mathematical modeling that eventually divides the liver into the eight subregions of Couinaud. The methodology was tested to segment the liver using 20 different exams and five different measures of performance, and the results obtained confirm the potential of the method. The cases in which the method presented a poor performance are also discussed in order to instigate further research.

\section{Keywords}

Medical Imaging. Segmentation. Level Sets. Genetic Algorithms. Computer Tomography. 


\section{Sumário}

1 Introdução $\quad 11$

1.1 Motivação 12

1.2 Objetivos 13

$\begin{array}{lll}1.3 & \text { Estrutura da Dissertação } & 15\end{array}$

2 Imagens Médicas e Anatomia do Fígado $\quad 17$

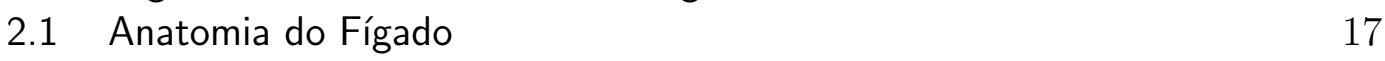

$\begin{array}{ll}2.2 \text { Tomografia Computadorizada } & 19\end{array}$

2.2.1 Padrão DICOM 22

3 Fundamentos Teóricos $\quad 23$

3.1 Imagens Digitais 23

3.2 Segmentação de Imagens 24

3.2.1 Segmentação Orientada a Dados 25

3.2.2 Segmentação Orientada a Modelo 27

3.3 Level Sets 27

3.3.1 Superfícies Implícitas 28

3.3.2 Métodos de Level Sets 31

3.4 Algoritmos Genéticos 33

3.4.1 Fundamentos 33

3.4.2 Representação 34

3.4.3 Avaliação e Seleção 35

3.4.4 Operadores Genéticos 37

4 Modelagem da Segmentação $\quad 40$

4.1 Segmentação do Fígado 40

4.1.1 Modelagem do método 40

4.1.2 Otimização dos parâmetros de segmentação 44

4.2 Segmentação dos Vasos e Nódulos 48

4.2.1 Modelo de Mistura 49

4.2.2 Segmentação de Vasos e Nódulos a partir do Modelo de Mistura 51

4.3 Identificação das Veias Porta e Hepática 52

4.4 Segmentação das Regiões de Couinaud $\quad 54$

5 Avaliação Experimental $\quad 57$

5.1 Protótipos $\quad 57$

$\begin{array}{lll}\text { 5.1.1 3DLiver } & 57\end{array}$

5.1.2 Level Sets AG $\quad 59$

5.2 Avaliação de resultados $\quad 60$

5.2.1 Segmentação do Fígado $\quad 61$

5.2.2 Segmentação de Vasos e Nódulos 63

5.2.3 Identificação de Veias 63

5.2.4 Segmentação das Regiões de Couinaud 64 
6 Conclusões $\quad 72$

6.1 Avaliação do Trabalho $\quad 72$

$\begin{array}{lll}6.2 & \text { Trabalhos Futuros } & 73\end{array}$

$\begin{array}{lll}6.3 & \text { Considerações Finais } & 73\end{array}$

$\begin{array}{lr}\text { Referências Bibliográficas } & \mathbf{7 5}\end{array}$

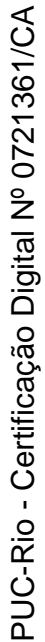




\section{Lista de figuras}

2.1 Localização do Fígado. 18

2.2 Anatomia Morfológica do Fígado: lobos esquerdo e direito. 19

2.3 Anatomia Funcional do Fígado: lobos esquerdo e direito. 19

2.4 Anatomia Funcional do Fígado: segmentos de Couinaud. 20

2.5 Tomografia Computadorizada da região abdominal. 21

3.1 Imagem tridimensional. 24

3.2 Processo evolucionário. $\quad 35$

3.3 Seleção por roleta. 36

3.4 Crossover de um ponto. 38

3.5 Crossover de dois pontos. 39

3.6 Mutação. 39

4.1 Definição de $P(x)$ baseando-se em limiares. $\quad 42$

4.2 Definição dos limiares $T L$ e $T H$. 43

4.3 Avaliação da aptidão de cada indivíduo. 45

4.4 Mistura de gaussianas: $G_{e}$ em verde, $G_{c}$ em azul, $G_{d}$ em vermelho e $\mathrm{Hi}$ em preto. $\quad 49$

4.5 Intervalos para segmentação de nódulos e vasos por histerese. $\quad 55$

4.6 Identificação de veias hepática esquerda, média e direita. 56

5.1 3DLiver - Tela inicial. 58

5.2 3DLiver - Módulos de segmentação (a), visualização (b) e edição (c). 59

5.3 Level Sets AG - Otimização de Parâmetros. 60

5.4 Melhor resultado obtido: vista axial, vista coronal, vista sagital. $\quad 65$

5.5 Fígado com um grande nódulo: vista axial, vista coronal, vista sagital. 66

5.6 Segmentação de vasos (em vermelho) e nódulos (em verde escuro): $\begin{array}{ll}\text { vista axial, vista coronal, vista sagital. } & 67\end{array}$

5.7 Segmentação de vasos e nódulos: modelo 3D. 68

5.8 Identificação das veias: ramos principais da veia hepática. 68

5.9 Identificação das veias: veias hepática (vermelho) e porta (azul). 69

5.10 Segmentos de Couinaud em diferentes cores: vista axial, vista coronal, vista sagital. $\quad 70$

5.11 Segmentos de Couinaud em diferentes cores. 71 


\section{Lista de tabelas}

5.1 Tabela de resultados da segmentação do fígado.

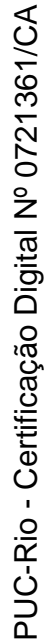

